\title{
Assessing the genetic relationships of Curcuma alismatifolia varieties using simple sequence repeat markers
}

\author{
S. Taheri ${ }^{1}$, T.L. Abdullah ${ }^{1}$, N.A.P. Abdullah ${ }^{1}$, Z. Ahmad ${ }^{2}$, E. Karimi ${ }^{1}$ and \\ M.R. Shabanimofrad ${ }^{1}$ \\ ${ }^{1}$ Department of Crop Science, Faculty of Agriculture, \\ University Putra Malaysia, Serdang, Selangor, Malaysia \\ ${ }^{2}$ Agrotechnology and Biosciences Division, \\ Malaysian Nuclear Agency (Nuclear Malaysia), Bangi, Selangor, Malaysia \\ Corresponding author: S. Taheri \\ E-mail: sima_taheri65@yahoo.com \\ Genet. Mol. Res. 13 (3): 7339-7346 (2014) \\ Received June 28, 2013 \\ Accepted July 5, 2014 \\ Published September 5, 2014 \\ DOI http://dx.doi.org/10.4238/2014.September.5.12
}

\begin{abstract}
The genus Curcuma is a member of the ginger family (Zingiberaceae) that has recently become popular for use as flowering pot plants, both indoors and as patio and landscape plants. We used PCR-based molecular markers (SSRs) to elucidate genetic variation and relationships between five varieties of Curcuma (Curcuma alismatifolia) cultivated in Malaysia. Of the primers tested, 8 (of 17) SSR primers were selected for their reproducibility and high rates of polymorphism. The number of presumed alleles revealed by the SSR analysis ranged from two to six alleles, with a mean value of 3.25 alleles per locus. The values of $H_{\mathrm{O}}$ and $H_{\mathrm{E}}$ ranged from 0 to 0.8 (mean value of 0.2 ) and 0.1837 to 0.7755 (mean value of 0.5102 ), respectively. Eight SSR primers yielded 26 total amplified fragments and revealed high rates of polymorphism among the varieties studied. The polymorphic information content varied from 0.26 to 0.73 . Dice's similarity coefficient was calculated for all pairwise comparisons and used to construct an unweighted pair group method with arithmetic average (UPGMA) dendrogram. Similarity coefficient values
\end{abstract}


from 0.2105 to 0.6667 (with an average of 0.4386 ) were found among the five varieties examined. A cluster analysis of data using a UPGMA algorithm divided the five varieties/hybrids into 2 groups.

Key words: Curcuma alismatifolia; Genetic relationships; Molecular markers; Simple sequence repeats

\section{INTRODUCTION}

Curcuma alismatifolia is a member of the Zingiberaceae family, which originates from the tropical and subtropical areas of northern Thailand and Cambodia (Apavatjrut et al., 1996). C. alismatifolia has recently become popular in the ornamental flower market because of their colorful, stiff, and long-lasting inflorescences, with pink-purplish coma bracts in the upper part and green coma bracts in the lower part. Small true flowers bear inside the bracts (Khuankaew et al., 2010). Traditional methods based on phenotypic observations for identifying Curcuma varieties in Curcuma spp. are slow and have limitations. However, new methods based on studies of DNA variations to assess genetic relationships or diversity among varieties can accelerate ornamental plant breeding programs. Molecular markers have become useful for breeding and cultivar development in many crops (Syamkumar and Sasikumar, 2007). Among the molecular markers available, microsatellites or simple sequence repeats (SSR), which are tandem repeats of 1-6 nucleotide long DNA motifs, have gained considerable importance in plant genetics and breeding because of their multi-allelic nature, codominant inheritance, high abundance, extensive genome coverage, reproducibility, and discriminatory power (Kalia et al., 2011). A variety of molecular marker techniques have been developed for measuring genetic variability/similarity in ornamental plants, such as random amplified polymorphic DNA (RAPD; Anthurium andraeanum and Chrysanthemum) (Nowbuth et al., 2005; Lapitan et al., 2007; Barakat et al., 2010), ISSR (Rose) (Jabbarzadeh et al., 2010), and SSR (Prunus mume and Nelumbonucifera) (Tian et al., 2008; Hayashi et al., 2008). Moreover, several molecular studies have employed various DNA markers in Curcuma spp. Apavatjrut et al. (1999) used isozyme markers to resolve the taxonomic confusion in the genus Curcuma. Sigrist et al. (2009) developed and characterized microsatellite markers (SSR) for Curcuma longa L. Das et al. (2011) used 12 RAPD, 19 ISSR, and 4 amplified fragment length polymorphism (AFLP) primers as molecular genetic fingerprints of 9 Curcuma spp. from northeast India, which were developed using polymerase chain reaction (PCR)-based markers. Taheri et al. (2012) used 16 ISSR primers to assess the genetic relationships of five $C$. alismatifolia varieties. They showed that the ISSR markers are more discriminating than those of RAPD and AFLP for evaluating the genetic diversity/relationships among Curcuma spp.

\section{MATERIAL AND METHODS}

\section{Source of plant materials}

The current study was conducted at the genetic and plant breeding laboratory in the Crop Science Department, Faculty of Agriculture, University Putra Malaysia. Five C. alismatifolia varieties, namely Chiang Mai Pink, Sweet Pink, Doi Tung 554, Chiang Mai Red, and Kimono Pink, were purchased from the Curcuma Nursery (Ubonrat) in Doisaket District, Chiang Mai 50220, Thailand. 


\section{Total genomic DNA extraction}

Fully opened, fresh, and tender leaves of 25 samples of five varieties/hybrids of $C$. alismatifolia were used for the isolation of DNA. Approximately $0.1 \mathrm{~g}$ young leaf tissue was ground in liquid nitrogen into a fine powder and transferred to $2-\mathrm{mL}$ microcentrifuge tubes. The genomic DNA was isolated using $800 \mu \mathrm{L}$ modified cetyltrimethylammonium bromide (CTAB) extraction buffer (Doyle and Doyle, 1987). The extraction buffer was as follows: $2 \%$ (w/v) CTAB, $1.4 \mathrm{mM} \mathrm{NaCl}, 100 \mathrm{mM}$ Tris-HCL, pH 8.0, $20 \mathrm{mM}$ EDTA, 2\% (w/v) PVP, and $2 \%(\mathrm{v} / \mathrm{v}) \beta$-mercaptoethanol. The mixture was incubated at $65^{\circ} \mathrm{C}$ for $1 \mathrm{~h}$. The extraction was followed by the addition of $600 \mu \mathrm{L}$ chloroform/isoamyl alcohol [24:1 (v/v)], and the resultant supernatants were transferred to new microcentrifuge tubes. Ethanol $(95 \%)$ was used to precipitate nucleic acids, and the samples were kept at $-20^{\circ} \mathrm{C}$ overnight. The pellet obtained was dissolved in a Tris-EDTA (TE) buffer ( $10 \mathrm{mM}$ Tris- $\mathrm{HCl}, \mathrm{pH}=8.0$, and $1 \mathrm{mM}$ EDTA, $\mathrm{pH}$ $=8.0$ ). Coprecipitated RNA was removed by digestion with RNase. The concentration and purity of isolated DNA was determined using the NanoDrop 2000 (Thermo Fisher Scientific Inc.), and the quality was verified by electrophoresis on $0.8 \%$ agarose gel.

\section{PCR amplification and product electrophoresis}

PCR amplification was carried out for eight SSR primers in a $25-\mu \mathrm{L}$ reaction volume. The PCR reaction contained $70 \mathrm{ng} / \mu \mathrm{L}$ genomic DNA, 2X DreamTaq ${ }^{\mathrm{TM}}$ Green PCR Master Mix (Fermentas, International Inc.), and $0.4 \mu \mathrm{M}$ forward and reverse primers. Amplification was performed in a thermal cycler (Bio-Rad Laboratories, Inc.) for a total of 40 cycles after an initial denaturation of the template DNA at $94^{\circ} \mathrm{C}$ for $3 \mathrm{~min}$. This was followed by 10 cycles of $94^{\circ} \mathrm{C}$ for $40 \mathrm{~s}$, a touch-down one-degree decrement for annealing temperature starting at $7^{\circ} \mathrm{C}$ above Tm for each primer for $30 \mathrm{~s}$ and $72^{\circ} \mathrm{C}$ for $1 \mathrm{~min}$. This was followed by 30 cycles of $95^{\circ} \mathrm{C}$ for $40 \mathrm{~s}$, a final annealing temperature for $30 \mathrm{~s}$ and $72^{\circ} \mathrm{C}$ for $1 \mathrm{~min}$, and a final extension of $72^{\circ} \mathrm{C}$ for $10 \mathrm{~min}$. The amplification products were analyzed on $4 \%$ metaphor gel with a 50-bp DNA ladder (Ready-to-use). The gel was stained with Midori green, visualized under ultraviolet light, and photographed by a gel documentation system (ChemilImager ${ }^{\mathrm{TM}}$ Gel Doc., Alpha Innotech Corporation, San Jose, CA, USA).

\section{Data scoring and analysis}

In the case of SSR, each band was considered a separate putative locus. Only clear, unambiguous, and reproducible bands were considered for data analysis. Each band was considered a single locus. The presence or absence of each single fragment was coded by 1 or 0 , respectively, and scored for a binary data matrix. The binary data matrix was entered into the Numerical Taxonomy and Multivariate Analysis System (NTSYSpc 2.10e) (Rohlf, 2002). The level of similarity among species was established as the percentages of polymorphic bands and a matrix of genetic similarity compiled using Dice's similarity index (Dice, 1945). Similarity coefficients were used to construct the dendrogram using the unweighted pair group method with arithmetic average (UPGMA) and the sequential hierarchical and nested clustering (SHAN) routine in the NTSYSpc program, thus representing the genetic relationship among five Curcuma varieties. Measurement of diversity, including $H_{\mathrm{O}}, H_{\mathrm{E}}(\mathrm{Nei}, 1973), N_{\mathrm{A}}$, the effective number of alleles $\left(N_{\mathrm{E}}\right)$, Shannon's information 
index (I), and the percentage of polymorphic bands (PPBs), were estimated using the POPGENE 1.31 software (Yeh et al., 1997). It was assumed that the gene frequency within a population was in Hardy-Weinberg equilibrium. To compare the efficiency of the primers, the polymorphic information content (PIC) as a marker discrimination power was calculated according to Cordeiro et al. (2000) using a PIC calculator (http://www.liv.ac.uk/ kempsj/pic.html). The calculation was based on the number of alleles per locus.

\section{RESULTS AND DISCUSSION}

\section{SSR amplification}

Of the 17 primer pairs, 9 showed monomorphic products. The remaining 8 primer pairs resulted in polymorphic and readable PCR products (Figure 1A-D). Table 1 summarizes the results obtained based on the analysis of five Curcuma varieties/hybrids using the polymorphic SSR loci. The $N_{\mathrm{A}}$ varied widely among these loci. The $N_{\mathrm{A}}$ ranged from 2 (clon04, clon09, clon11, and clon14) to 6 (clon12), with an average value of 3.25 per locus. On a per locus basis, these numbers were smaller than the average values of 6.6 and 6.7 (ranging from 2 to 11) alleles per locus for various classes of microsatellites reported by Sigrist et al. (2009).
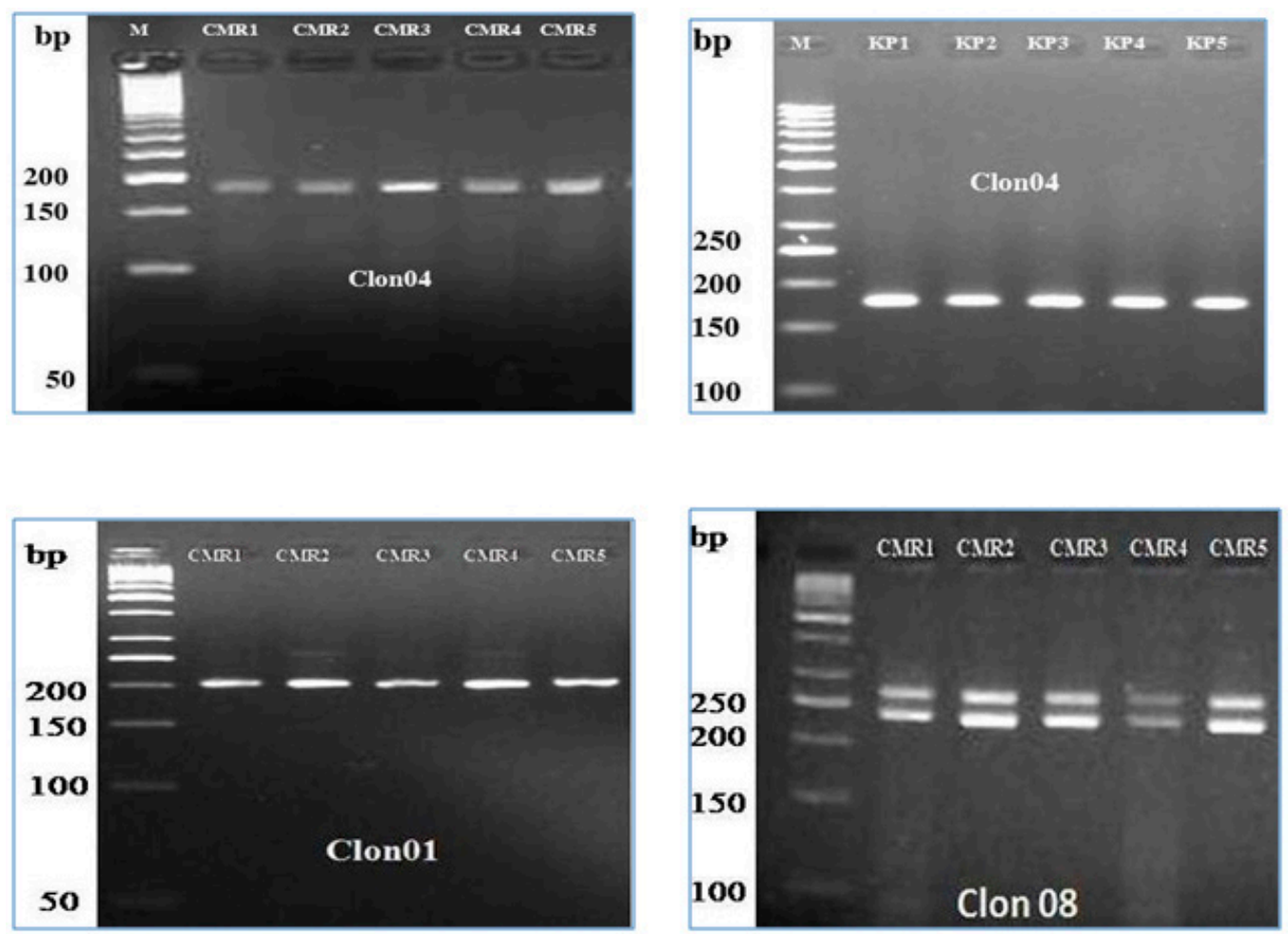

Figure 1. Banding pattern of different Curcuma alismatifolia varieties using simple sequence repeat (SSR) markers. Lane $M=$ Marker; $\mathrm{CMR}=$ Chiang Mai Red; KP = Kimono Pink. 
Genetic relationships of C. alismatifolia using SSR markers

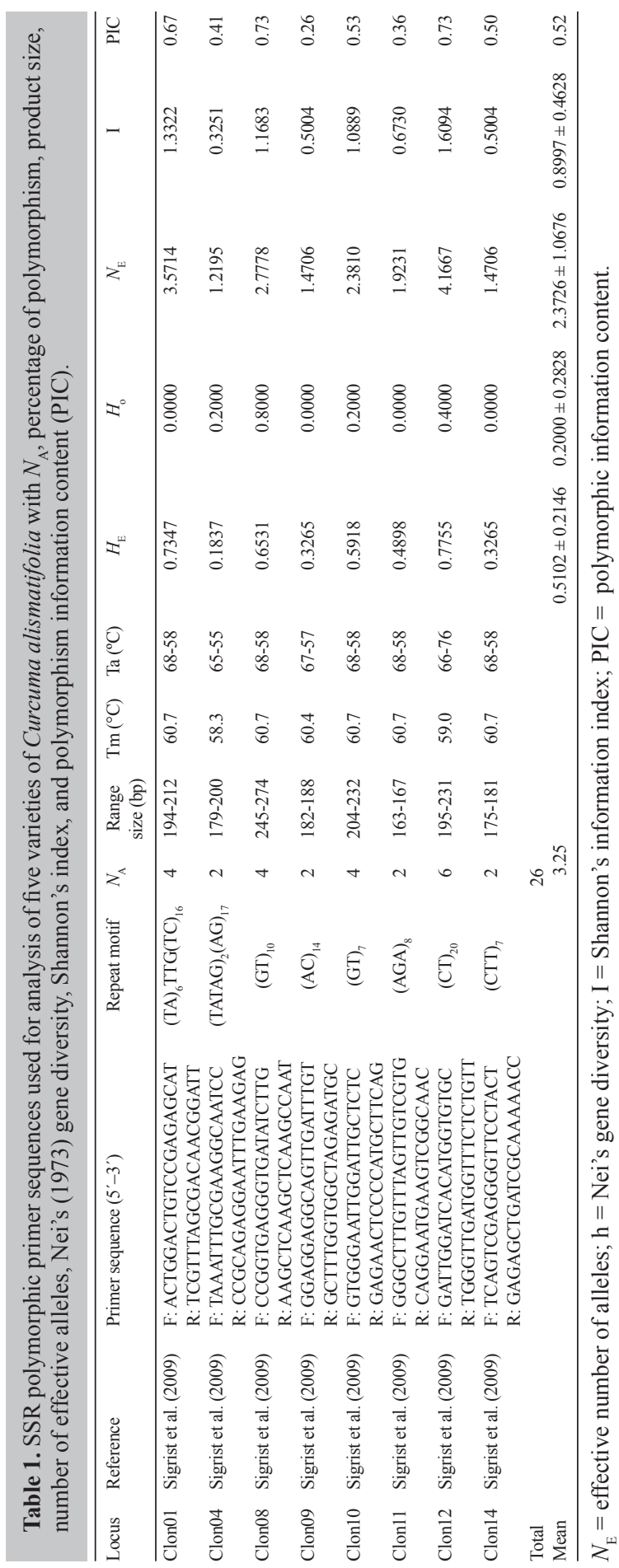


The average $N_{\mathrm{A}}$ per locus obtained in the study was also smaller than that reported in previous studies using other types of markers such as RAPD, ISSR, and AFLP (Syamkumar and Sasykumar, 2007; Das et al., 2011), and allozymes (Paisooksantivatana et al., 2001). The $N_{\mathrm{E}}$ per locus ranged from 1.2 (clon04) to 3.5 (clon01), with an average of 2.3. The difference between the average $N_{\mathrm{A}}$ and $N_{\mathrm{E}}$ was due to the uneven frequency of each allele (Liao et al., 2011). PIC provides an estimate of the discriminatory power of a marker to differentiate genotypes based on both the $N_{\mathrm{A}}$ expressed and their relative frequencies (Nagl et al., 2011). The PIC value, which measures allele diversity and frequency among varieties, also varied from one locus to another. The PIC value was 0.52 per marker and ranged from 0.26 (clon09) to 0.73 (clon08 and clon12), indicating that most loci were highly polymorphic and informative. The genetic diversity of each SSR locus appeared to be associated with the $N_{\mathrm{A}}$ detected per locus. The smaller the PIC value of a locus, the fewer number of alleles detected. This pattern observed was consistent with that reported by Sigrist et al. (2009). Using Shannon's diversity index, an overall genetic diversity of 0.8997 was obtained from the analysis, indicating a relatively high level of genetic variation among studied varieties. The higher value of I represents the effectiveness of microsatellite loci to detect variation (Babaei et al., 2012). In this study, loci amplifying dinucleotide repeat motifs were found to be more polymorphic, with an average value of 5 alleles, than those with trinucleotide or tetra nucleotide repeat motifs, which both yielded an average value of two alleles. Among the loci with perfect or compound dinucleotide repeat motifs, the marker with the CT repeat motif showed the greatest variability. These results suggest that the total repeat count of SSR loci is associated with $N_{\mathrm{A}}$ (Lapitan et al., 2007). In this study, the greater the repeat number in the microsatellite DNA, the greater the $N_{\mathrm{A}}$ identified. The estimates of genetic similarity ranged from $21 \%$ for the most distant varieties (i.e., Sweet Pink and Kimono Pink) to $0.67 \%$ between Chiang Mai Red and Chiang Mai Pink (Table 2).

$\begin{aligned} & \text { Table 2. Genetic similarity indices between each pair of the five Curcuma varieties (C. alismatifolia) based } \\
& \text { on SSR fragment analysis. }\end{aligned}$
\begin{tabular}{lcccc}
\hline Varieties & Chiang Mai Pink & Sweet Pink & Doi Tung 554 & Chiang Mai Red \\
\hline Sweet Pink & 0.6000 & & & \\
Doi Tung 554 & 0.5263 & 0.2857 & 0.4211 & \\
Chiang Mai Red & 0.6667 & 0.5000 & 0.3333 & 0.2353 \\
Kimono Pink & 0.3529 & 0.2105 & &
\end{tabular}

\section{Clustering of Curcuma varieties}

Genetic similarity values among the Curcuma varieties led to the construction of a dendrogram (Figure 2). The UPGMA dendrogram showed two main clusters at a coefficient level of 0.38. The first one included only the Kimono Pink variety and yielded a Dice's similarity index of 0.28 in comparison to other varieties, while the second included the other four varieties. In the second cluster, there were two subclusters. The first subcluster included Doi Tung 554; Sweet Pink, Chiang Mai Red, and Chiang Mai pink were assigned to the second subcluster. A comparison of Chiang Mai Red and Chiang Mai Pink yielded the maximum observed similarity value (0.67 DSI). 


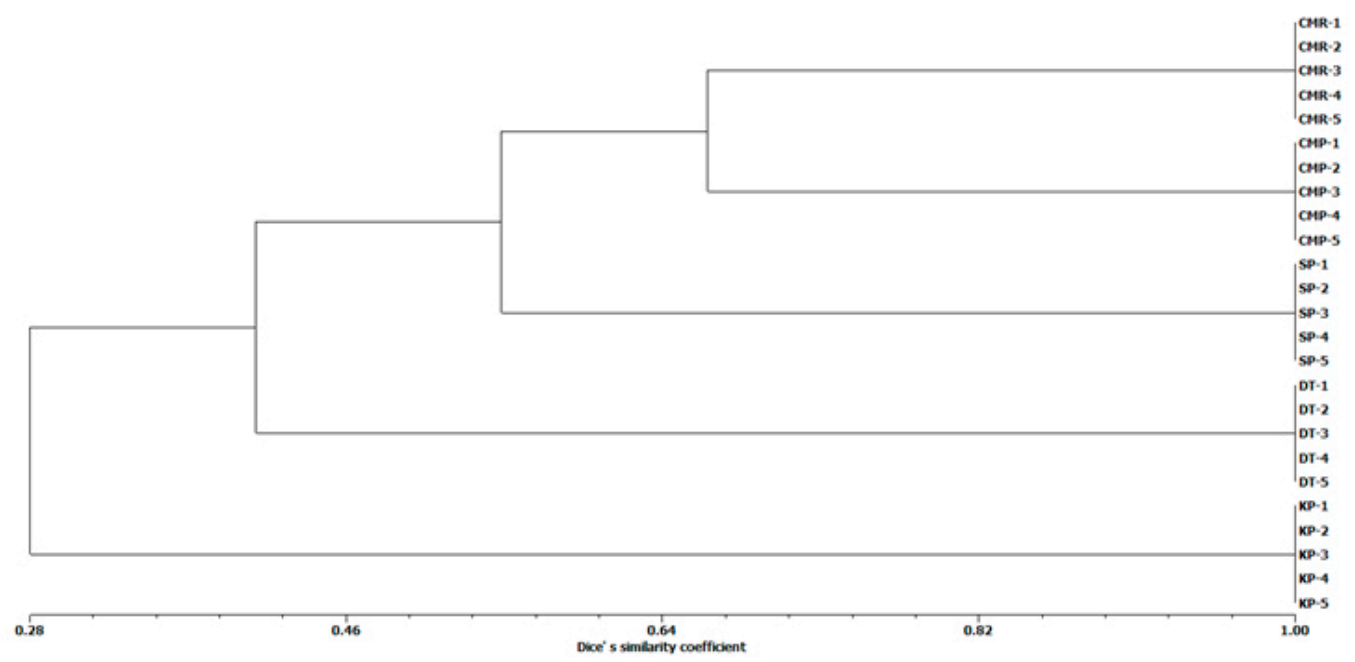

Figure 2. Dendrogram demonstrating the relationships among the 5 Curcuma alismatifolia varieties based on SSRs.

\section{CONCLUSION}

In summary, this study provided an overview of the genetic relationship of the $C$. alismatifolia varieties/hybrids. The use of SSR markers in genetic relationship analyses enabled the varieties to be grouped according to their genetic similarities/distances. Although there have been previously published reports on the use of other molecular marker techniques, such as RAPD, AFLP, and ISSR, for analyzing various species of Curcuma, the codominant nature of SSRs allows them to be used as a powerful tool for assessing the genetic diversities/ relationships of the varieties under study. Additionally, the results obtained from this study confirm the complete similarity of different samples from each variety, and these results would be useful for improved management and identification of $C$. alismatifolia varieties for future breeding programs such as mutation breeding.

\section{ACKNOWLEDGMENTS}

Research supported by the fundamental research grant scheme (FRGS) under the Ministry of Higher Education in Malaysia.

\section{REFERENCES}

Apavatjrut P, Sirisawad T, Sirirugsa P and Voraurai P (1996). Studies on Chromosome Number of Seventeen Thai Curcuma Species. Proceedings of the 2nd Thailand National Ornamental Meeting, Chiang Mai.

Apavatjrut P, Somboon A, Puangpen S and Chiara A (1999). Molecular markers in the identification of some early flowering Curcuma L. (Zingiberaceae) species. Ann. Bot. 84: 529-534.

Babaei N, Abdullah NA, Saleh G and Abdullah TL (2012). Isolation and characterization of microsatellite markers and analysis of genetic variability in Curculigo latifolia Dryand. Mol. Biol. Rep. 39: 9869-9877.

Barakat MN, Abdel Fattah RS, Badr M and El-Torky MG (2010). In vitro mutagenesis and identification of new variants 
via RAPD markers for improving Chrysanthemum morifolium. Afr. J. Agri. Res. 8: 748-757.

Cordeiro GM, Taylor GO and Henry RJ (2000). Characterisation of microsatellite markers from sugarcane (Saccharum sp.), a highly polyploid species. Plant Sci. 155: 161-168.

Das A, Kesari V, Satyanarayana VM, Parida A, et al. (2011). Genetic relationship of Curcuma species from Northeast India using PCR-based markers. Mol. Biotechnol. 49: 65-76.

Dice LR (1945). Measures of the amount of ecologic association between species. Ecology 26: 297-302.

Doyle JJ and Doyle JL (1987). A rapid DNA isolation procedure for small quantities of fresh leaf tissue. Phytochem. Bull. 19: 11-15.

Hayashi K, Shimazu K, Yaegaki H, Yamaguchi M, et al. (2008). Genetic diversity in fruiting and flowers-ornamental Japanese apricot (Prunus mume) germplasms assessed by SSR markers. Breed. Sci. 58: 401-410.

Jabbarzadeh Z, Khosh-khui M, Salehi H and Saberivand A (2010). Inter simple sequence repeat (ISSR) markers as reproducible and specific tools for genetic diversity analysis of rose species. Afr. J. Biotechnol. 9: 6091-6095.

Kalia RK, Rai MK, Kali S, Singh R, et al. (2011). Microsatellite markers: an overview of the recent progress in plants. Euphytica 177: 309-334.

Khuankaew T, Ruamrungsri S, Ito S, Sato T, et al. (2010). Assimilation and translocation of nitrogen and carbon in Curcuma alismatifolia Gagnep. Plant Biol. 12: 414-423.

Lapitan VC, Brar DS, Abe T and Redona ED (2007). Assessment of genetic diversity of Philippine rice cultivars carrying good quality traits using SSR markers. Breed. Sci. 57: 263-270.

Liao M, Wang Y, Rong X, Zhang Z, et al. (2011). Development of new microsatellite DNA markers from Apostichopus japonicus and their cross-species application in Parastichopus parvimensis and Pathallus mollis. Int. J. Mol. Sci. 12: $5862-5870$.

Nagl N, Taski-Ajdukovic K, Popovic A and Curcic A (2011). Estimation of genetic variation among related sugar beet genotypes by using RAPD. Genetika 43: 575-582.

Nei M (1973). Analysis of gene diversity in subdivided populations. Proc. Natl. Acad. Sci. U. S. A. 70: 3321-3323.

Nowbuth P, Khittoo G, Bahorun T and Venkatasamy S (2005). Assessing genetic diversity of some Anthurium andraeanum Hort. cut-flower cultivars using RAPD Markers. Afr. J. Biotech. 4: 1189-1194.

Paisooksantivatana Y, Kako S and Seko H (2001). Genetic diversity of Curcuma alismatifolia Gagnep. (Zingiberaceae) in Thailand as revealed by allozyme polymorphism. Genetic Res. Crop Evol. 48: 459-465.

Rohlf FJ (2002). NTSYS-pc: Numerical Taxonomy System, Version 2.1. Exeter Publishing, Ltd., Setauket.

Sigrist MS, Pinheiro JB, Azevedo-Filho JA and Colombo CA (2009). Development and characterization of microsatellite markers for turmeric (Curcuma longa). Plant Breed. 129: 570-573.

Syamkumar S and Sasikumar B (2007). Molecular marker based genetic diversity analysis of Curcuma species from India. Sci. Hort. 112: 235-241.

Taheri S, Abdullah TL, Abdullah NA and Ahmad Z (2012). Genetic relationships among five varieties of Curcuma alismatifolia (Zingiberaceae) based on ISSR markers. Genet. Mol. Res. 11: 3069-3076.

Tian HL, Chen XQ, Wang JX and Xue JH (2008). Development and characterization of microsatellite loci for lotus (Nelumbo nucifera). Conserv. Genet. 9: 1385-1388.

Yeh FC, Yang R, Boyle TBJ and Ye Z (1997). POPGENE, The User-Friendly Shareware for Population Genetic Analysis, Molecular Biology and Biotechnology Centre. University of Alberta, Edmonton. 